\title{
How insignificant modifications of photocatalysts can significantly change their photocatalytic activity
}

\section{Trochowski, Mateusz}

2019-11-21

Trochowski , M , Kobielusz, M , Mroz , K, Surowka , M K, Hämäläinen , J , livonen , T, Leskelä , M \& Macyk , W 2019 , ' How insignificant modifications of photocatalysts can significantly change their photocatalytic activity ' , Journal of Materials Chemistry. A, vol. 7 , no. 43 , pp. 25142-25154 . https://doi.org/10.1039/c9ta09400h

http://hdl.handle.net/10138/321780

https://doi.org/10.1039/c9ta09400h

acceptedVersion

Downloaded from Helda, University of Helsinki institutional repository.

This is an electronic reprint of the original article.

This reprint may differ from the original in pagination and typographic detail.

Please cite the original version. 


\title{
How Insignificant Modifications of Photocatalysts Can Significantly Change their Photocatalytic Activity
}

Received 00th January 20xx, Accepted 00th January 20xx DOI: $10.1039 / x 0 \times x 00000 x$

\author{
Mateusz Trochowski, ${ }^{a}$ Marcin Kobielusz, ${ }^{a *}$ Krystian Mróz, ${ }^{a}$ Marcin Surówka, ${ }^{a}$ Jani Hämäläinen, ${ }^{b}$ \\ Tomi livonen, ${ }^{\mathrm{b}}$ Markku Leskeläb ${ }^{\mathrm{b}}$ and Wojciech Macyk $^{\mathrm{a} *}$
}

\begin{abstract}
Synthetic procedures, including doping, sintering and surface coating can noticeably affect physicochemical properties of semiconductors. Introduced changes very often translate to the photocatalytic and photoelectrochemical activity alterations. However, in this work we have focused on more subtle treatments, which result in lack of changes observed using XRD, UV-vis, porosimetry, TEM or SEM. We have exposed titanium dioxide (P25, UV100) to a treatment with reducing agents used in procedures of noble metals deposition (citrate, borohydride, photoreduction), or surface decoration with small amounts of $\mathrm{TiO}_{2}$ by atomic layer deposition (ALD; 10 to 200 deposition cycles), which presumably should be neutral to its activity. Although the "classical" characterization methods did not show any differences between original and treated samples, spectroelectrochemical (SE-DRS) determination of the density of states (DOS) and catechol adsorption tests revealed a significant influence of such treatments on photocatalytic activity (photogeneration of $\mathrm{HO}^{\bullet}$ radicals, water reduction, herbicides degradation) and photoelectrochemical behaviour of the studied samples. We have shown, that the applied slight surface modifications of titanium dioxide ("insignificant" at the first glance) may strongly affect the activity of this material. Such often overlooked effects must be taken into account during a comparative photoactivity analysis of various semiconductors, since an insignificant surface treatment may noticeably influence surface chemistry. We have also demonstrated that SE-DRS can be considered as a useful tool to study these effects, although it can be difficult to correlate a particular treatment with recorded changes in the density of states.
\end{abstract}

\section{Introduction}

For almost fifty years titanium(IV) oxide has been intensively investigated and became one of the most popular photocatalysts. The reasons for this is its non-toxicity, chemical stability, cheapness and impressive photocatalytic properties. ${ }^{1}$ Nevertheless, $\mathrm{TiO}_{2}$ has a relatively wide bandgap $(\sim 3.2 \mathrm{eV}$ for anatase, $\sim 3.0 \mathrm{eV}$ for rutile), thus it can harvest only about $5 \%$ of natural solar light. ${ }^{2}$ Broadening its absorption range is highly desirable. ${ }^{3}$ Mentioned limitation leads to many strategies that can affect $\mathrm{TiO}_{2}$ morphology (increasing pore size or specific surface area) or chemical composition (enrichment of titanium

\footnotetext{
a. Faculty of Chemistry, Jagiellonian University, ul. Gronostajowa 2, 30-387 Kraków, Poland; e-mail: kobielusz@chemia.uj.edu.pl,macyk@chemia.uj.edu.pl

b. University of Helsinki, Department of Chemistry, Laboratory of Inorganic Chemistry, P.O. Box 55, Fl-00014 Helsinki, Finland

Electronic Supplementary Information (ESI) available: Exemplary plot of $\mathrm{TAOH}$ formation upon the visible light irradiation, plots of hydrogen formation upon the UV light irradiation, XRD patterns of investigated $\mathrm{TiO}_{2}$ samples, specific surface area values, pore size and pore volume of investigated material, amounts of catecho adsorbed at the surface of the studied materials modified with ALD, diffuse reflectance spectra of surface modified $\mathrm{TiO}_{2}$, representative density of states patterns for $\mathrm{TiO}_{2}$ after ALD application, diffuse reflectance spectra of $\mathrm{TiO}_{2}$ materials decorated with gold nanoparticles, XRD patterns of investigated $\mathrm{TiO}_{2}$ sample synthesized in the presence of gold ions with marked Au reflexes, EDS analysis for $\mathrm{TiO}_{2}$ materials synthesized in the presence of gold, diffuse reflectance spectra of $\mathrm{TiO}_{2}$ materials without gold, XRD patterns of investigated $\mathrm{TiO}_{2}$ synthetized in the absence of gold ions, representative density of states patterns for $\mathrm{TiO}_{2}$ after preparation without gold precursor, amounts of catechol adsorbed at the surface of materials modified with gold deposition procedures. See DOI: 10.1039/x0xx00000x
}

dioxide structure by additional elements). The most common methods used to influence the absorption range includes nonmetal doping (by nitrogen, fluorine, sulfur, carbon and oxygen), noble and transition metals deposition or synthesis of coupled semiconductors. ${ }^{4-6}$ Another approaches include attachment of organic dyes at the surface of semiconductor ${ }^{7}$ or synthesis of reduced $\mathrm{TiO}_{2}$ materials $\left(\mathrm{TiO}_{2-x}\right) .{ }^{8}$

All of enlisted methods have some pros and cons toward improving photocatalytic properties of $\mathrm{TiO}_{2}$. Non-metal doping can broaden the absorption range and improve thermal stability of titanium(IV) oxide crystal lattice. ${ }^{3}$ However, these inorganic ions, due to their weak visible light absorption cannot harvest photons sufficiently. ${ }^{9}$ In case of transition metals their dopants can lead to increment of $\mathrm{Ti}^{3+}$ ions in crystalline titania matrix. It also enables formation of intrabandgap states close to the conduction band (CB) or valence band (VB) edges, useful in visible light utilization. ${ }^{3}$ Unfortunately, these materials are often thermally unstable and prone to carrier recombination. ${ }^{9}$ Moreover, introduced cations can create deep, localized $d$ states which could become undesirable recombination centers. ${ }^{10}$ Deposition of gold nanoparticles (GNps) is commonly used to enhance titanium(IV) oxide absorption range due to generation of surface plasmons. GNps also act as electron sinks for $\mathrm{TiO}_{2}$ electrons photogenerated in $\mathrm{CB}$, boosting separation of electron - hole pair and their lifetimes. ${ }^{11}$ However, Au-based materials are rather expensive and they suffer from impecunious thermal stability. ${ }^{9}$ Coupled semiconductors 
become more popular because of their enhanced charge separation. Lifetime of charge carriers in such systems can be efficiently prolonged through appropriate band edge positioning that are thermodynamically favorable. ${ }^{3}$ Regrettably, some very promising semiconductors, that can be coupled with $\mathrm{TiO}_{2}$ (like CdS), suffer from serious photocorrosion and rather fast recombination of charge carriers. ${ }^{12}$ Sensitization of a semiconductor with organic molecules is possible via two main approaches: adsorption of organic dye on the titania surface that leads to an indirect electron injection, or the chargetransfer formation between dye and $\mathrm{TiO}_{2}$ enabling a direct electron injection. ${ }^{13}$ Both methods broaden the absorption range of such sensitized semiconductor to the visible light and enhance their photoactivity. ${ }^{14}$ These materials are affordable and easy to obtain, but they are prone to oxidation processes. Even though their degradation products may undergo autocatalytic hydroxylation processes and favor the visible light utilization through the $\mathrm{HOMO} \rightarrow \mathrm{CB}$ electron transfer route, ${ }^{15}$ they are not commonly used in photocatalysis. Moreover, dye molecules used as sensitizers can desorb from the semiconductors surface and decrease photoactivity. ${ }^{9}$ Reduced $\mathrm{TiO}_{2}$ materials $\left(\mathrm{TiO}_{2-x}\right)$ gain recently growing a lot of attention because of their capabilities of flexible structural changes (surface or bulk) and approachable range of colors. They reveal extended absorption of solar light resulting in high photocatalytic and photoelectrochemical activity. ${ }^{8}$ Unfortunately, reduction of titanium dioxide may introduce electronic states below the conduction band minimum resulting in poor electron mobility in the $\mathrm{TiO}_{2}$ bulk. ${ }^{16}$

The effectiveness of used modifications is usually verified by comparison of photocatalytic activity of modified and original samples. Very often positive or negative effects are attributed only to the modifier itself. For instance, in the case of different techniques of gold nanoparticles deposition at the $\mathrm{TiO}_{2}$ surface photoactivity changes are usually attributed to the distribution of metal nanoparticles or crystal size and shape. The influence of different reducing agents on the photocatalyst surface and the resulting photoactivity of the material is rarely investigated. ${ }^{17}$ Usually effects related to synthesis conditions are neglected.

The activity of photocatalyst depends on lifetime, availability and redox potentials of the electron-hole pairs. ${ }^{18}$ All these factors can be affected by various surface or bulk defects of the crystal structure. Usually their presence is acknowledged as unfavorable for the photocatalytic processes. However, it has been reported that powder materials exhibit higher photoactivity and slower charge recombination even though they have more defects than defect-free single crystals of the same photocatalyst. ${ }^{19}$ Both bulk and surface defects can offer new electronic states, acting as electron or hole traps and - in the case of surface defects - reactive sites. ${ }^{20}$ Electronic states can be divided into shallow and deep ones based on their energy distance to the bands edges. The deep ones are usually regarded as unreactive because of less favorable redox potentials. ${ }^{21}$ Nevertheless, their presence can be beneficial. These defects are responsible for extending the lifetime of the electron-hole pairs. ${ }^{22}$ It happens in the case of rutile- $\mathrm{TiO}_{2}$, in which electrons can be trapped in deep electronic states prolonging the lifetime of holes and therefore increasing the probability of the multi-hole processes.

Kong et al. suggested, that in the case of nanocrystalline $\mathrm{TiO}_{2}$ a decrease in concentration ratio of bulk to surface defects significantly improves the electron-hole pair separation, leading to enhancement of the overall photoactivity. ${ }^{23}$ This effect is at least comparable or even more important than the influence of crystalline phase or exposed facets. ${ }^{20}$ Moreover, presence of deep and shallow traps can influence charge carriers behavior even more significantly than the crystal size or specific surface area. ${ }^{22}$ Therefore, it should be assumed that even small modifications of the photocatalyst surface structure can create new, different electronic states and affect the photocatalytic activity. In addition, widely used modifications or even treatment of photocatalysts can affect not only the absorption light range, but can also generate new and/or remove existing surface electronic states. In this paper we verify how significant changes in redox properties and photocatalytic activity can be induced by apparently insignificant surface modifications.

Atomic layer deposition (ALD) is one of the tools which can be used to modify surface of solids. This technique enables synthesis with an excellent reproducibility ${ }^{24}$ and allows building ultrathin and thin layers from different precursors. ${ }^{25}$ One ALD cycle consists of several steps in which the surface is exposed alternately with precursors followed by an inert gas purge. These stages can be repeated many times to obtain thicker films. ${ }^{26}$ Saturative surface reactions in ALD enable preparation of conformal and uniform thin films on both plane supports and powder materials. ${ }^{27}$ Drastic changes in underlying material can be avoided by appropriate choice of precursors and deposition temperature. However, crystallizing layers could constitute potential defects generating factors which influence the overall photocatalytic activity. The use of the same material, both as a substrate and a modifier (e.g., $\mathrm{TiO}_{2}$ deposited at $\mathrm{TiO}_{2}$ ), allows surface states alteration without introducing third elements. In this work we attempt to elucidate how apparently insignificant modifications introduced by ALD treatment can affect the overall activity of photocatalyst. In addition, we will compare these effects with those induced by GNp synthesis conditions to obtain a broader perspective on the influence of such subtle factors on photocatalytic activity.

\section{Experimental}

\subsection{Materials}

Two kinds of titanium(IV) oxide were used: Hombikat UV100, 67\% anatase, $300 \mathrm{~m}^{2} / \mathrm{g}$, (Sachtleben Chemie) and P25, anatase:rutile 78:14, $50 \mathrm{~m}^{2} / \mathrm{g}$, (Evonik). $\mathrm{TiO}_{2}$ has been modified using ALD procedure described below. Two herbicides $\left(2.5 \cdot 10^{-5} \mathrm{~mol} / \mathrm{dm}^{3}\right)$ were tested in the process of photocatalytic degradation: 2,4-D and 2,4,5-T (Sigma Aldrich). Redox properties of synthesized materials were examined in $0.1 \mathrm{~mol} / \mathrm{dm}^{3} \mathrm{LiClO}_{4}$ (Acros Organics) solution in anhydrous acetonitrile. 


\subsection{Atomic Layer Deposition}

Deposition of $\mathrm{TiO}_{2}$ on $\mathrm{TiO}_{2}$ was done using a Picosun SUNALE R-150 ALD reactor operated under a nitrogen atmosphere of approximately $10 \mathrm{mbar}$. Titanium tetraisopropoxide (TTIP, Aldrich, 97\%) and deionized water were used as precursors of $\mathrm{TiO}_{2}{ }^{28}$ All depositions were done at $150^{\circ} \mathrm{C}$ on the powder samples spread on glass plates $(10 \mathrm{~cm}$ diameter). The evaporation temperature for TTIP was $65^{\circ} \mathrm{C}$, while the water source was kept at room temperature. The pulsing sequence was comprised of $0.6 \mathrm{~s}$ TTIP $-1.0 \mathrm{~s} \mathrm{~N}_{2}$ purge $-0.6 \mathrm{~s}$ TTIP $30 \mathrm{~s} \mathrm{~N}_{2}$ purge $-0.6 \mathrm{~s} \mathrm{H}_{2} \mathrm{O}-1.0 \mathrm{~s} \mathrm{~N}_{2}$ purge $-0.6 \mathrm{~s} \mathrm{H}_{2} \mathrm{O}-30 \mathrm{~s} \mathrm{~N}_{2}$ purge. The double pulsing sequence was adopted to ensure that the precursor doses of both TTIP and $\mathrm{H}_{2} \mathrm{O}$ were sufficient for fully saturated coverage on the high surface area powder samples. The numbers of ALD deposition cycles applied to each powder sample were 10, 30, 50, 100 and 200.

\subsection{Gold deposition}

\subsubsection{Photoreduction procedure}

The synthesis was carried out in a similar manner to that described by Kmetykó et al. ${ }^{17} \mathrm{~A}$ reaction mixture containing a solution of methanol and water in a 1:1 ratio was prepared along with the addition of sodium citrate $\left(1.88 \cdot 10^{-4} \mathrm{~mol} / \mathrm{dm}^{3}\right)$, titanium dioxide and gold(III) chloride. The concentration of $\mathrm{AuCl}_{3}$ was chosen to reach $1 \%$ of $\mathrm{Au}: \mathrm{TiO}_{2}$ weight ratio. The mixture was deoxygenated with an argon stream and irradiated by $150 \mathrm{~W}$ xenon lamp with no additional filters for $2.5 \mathrm{~h}$. The material was then centrifuged and washed several times with water. The material was abbreviated as Au(Photo)@TiO 2 .

\subsubsection{Chemical reduction - procedure with $\mathrm{NaBH}_{4}$}

The syntheses were carried out according to the procedure described by Kmetykó et al. ${ }^{17}$ Trisodium citrate $\left(1.88 \cdot 10^{-4} \mathrm{~mol} / \mathrm{dm}^{3}\right)$ was used not only to reduce $\mathrm{HAuCl}_{4}$ but also to stabilize the forming $\mathrm{Au}$ nanoparticles. To the thermostated $\mathrm{TiO}_{2}$ suspension $\left(20^{\circ} \mathrm{C}\right)$ sodium citrate and gold(III) chloride were added to reach $1 \%$ of $\mathrm{Au}: \mathrm{TiO}_{2}$ weight ratio. Finally, freshly-prepared, ice-cold $\mathrm{NaBH}_{4}$ solution $\left(3 \cdot 10^{-3} \mathrm{~mol} / \mathrm{dm}^{3}\right)$ was added dropwise to the reaction mixture. The suspension turned purple immediately. After $1 \mathrm{~h}$ the suspension was washed with water and centrifuged several times. The material was abbreviated as $\mathrm{Au}\left(\mathrm{NaBH}_{4}\right) @ \mathrm{TiO}_{2}$.

\subsubsection{Chemical reduction - procedure with $\mathrm{C}_{3} \mathrm{H}_{4}(\mathrm{OH})(\mathrm{COONa})_{3}$}

The syntheses were carried out according to the Turkevich procedure. ${ }^{29}$ Titanium dioxide was suspended in an aqueous solution of sodium citrate and gold chloride (the molar ratio of citrate and gold was 10:1). The concentration of $\mathrm{AuCl}_{3}$ was chosen to reach $1 \%$ of $\mathrm{Au}: \mathrm{TiO}_{2}$ weight ratio. Then the reaction mixture was boiled at $100^{\circ} \mathrm{C}$ for $1 \mathrm{~h}$. After cooling, the suspension was washed with water and centrifuged several times. The material was abbreviated as Au(Citrate)@ $\mathrm{TiO}_{2}$.

\subsection{Characterization of Physicochemical Properties}

The X-ray diffraction patterns were recorded using a Rigaku MiniFlex 600. The X-ray diffraction data were collected in the step scan mode. The $\mathrm{X}$-ray $\mathrm{Cu} \mathrm{K}_{\alpha}$, radiation was applied and operated at $40 \mathrm{kV}, 15 \mathrm{~mA}$. The scanning speed was $3 \mathrm{deg} / \mathrm{min}$ at a step of $0.05 \mathrm{deg}$.

Nitrogen adsorption/desorption isotherms were measured at $77 \mathrm{~K}$ using a Micromeritics ASAP 2020 instrument. Specific surface areas were calculated using the Brunauer-EmmettTeller method within the relative pressure of $p / p_{0}=0.05-0.15$. Total pore volumes were calculated from the volumes of nitrogen adsorbed at $p / p_{0}=0.97$.

Diffuse reflectance spectra (DRS) were recorded at a UV-Vis-NIR spectrophotometer Shimadzu UV-3600, equipped with a $15 \mathrm{~cm}$ dia. integrating sphere, in the range of $250-800$ $\mathrm{nm}$. Obtained data were converted using the Kubelka-Munk (KM) function.

The scanning electron microscope (SEM) images were collected with VEGA 3 TESCAN with an $\mathrm{LaB}_{6}$ cathode. The transmission electron microscope (TEM) images were collected with Tecnai Osiris instrument (FEI) operating at $200 \mathrm{kV}$.

X-ray photoelectron spectra (XPS) of $\mathrm{TiO}_{2}$ samples were recorded at a Prevac photoelectron spectrometer equipped with a monochromatized aluminum source $\mathrm{AlK}_{\alpha}(E=1486.6 \mathrm{eV})$ and a hemispherical VG SCIENTA R3000 analyzer. The base pressure in the analysis chamber during the measurements was $5 \times 10^{-9}$ mbar. The low energy electron flood gun (FS40A-PS) was used to compensate the charge on the surface of nonconductive $\mathrm{TiO}_{2}$ samples.

\subsection{Redox properties of the materials}

Redox properties and density of states of the materials were determined using the spectroelectrochemical method developed recently in our laboratory. ${ }^{30,31}$ It is based on electrochemical measurements combined with UV-vis diffuse reflectance spectroscopy. All electrochemical measurements were carried out in the three-electrode setup with platinum wire and $\mathrm{Ag} / \mathrm{Ag}^{+}$electrode $\left[\mathrm{AgNO}_{3}\left(10 \mathrm{mmol} / \mathrm{dm}^{3}\right)\right.$ in $0.1 \mathrm{~mol} / \mathrm{dm}^{3} \mathrm{Bu}_{4} \mathrm{NClO}_{4}$ in acetonitrile] as the counter and reference electrodes, respectively. Materials deposited onto platinum foil ( $c a .1 \times 3 \mathrm{~cm}^{2}$ ) were used as working electrodes. The electrodes were placed in a cuvette with a quartz window filled with $0.1 \mathrm{~mol} / \mathrm{dm}^{3} \mathrm{LiClO}_{4}$ solution in acetonitrile. The cuvette was placed in front of the integrating sphere, facing the working electrode toward the light beam. Oxygen was thoroughly removed from the electrolyte by purging it with argon before (15 $\mathrm{min}$ ) and during experiments. The electrode potential was controlled by the electrochemical analyzer (Bio-Logic, SP-150). The applied potential was lowered every 10 $\mathrm{min}$ by $50 \mathrm{mV}$. The relative reflectance changes (at $780 \mathrm{~nm}$ ) were collected by PerkinElmer UV-vis Lambda 12 spectrometer equipped with a $5 \mathrm{~cm}$ diameter integrating sphere. The relative reflectance changes were converted to the Kubelka-Munk function $(\triangle K M)$. The density of states (DOS) was calculated as a difference in the Kubelka-Munk function between two consecutive potentials. 


\subsection{Photoelectrochemical measurements}

Photocurrents were measured using a three-electrode setup. Working electrode - the thin layer of the material - was prepared at the surface of ITO-coated transparent foil ( $60 \Omega$ /sq resistance, SigmaAldrich). A platinum wire and $\mathrm{Ag} / \mathrm{AgCl}$ were used as the counter and reference electrodes, respectively. The electrolyte $\left(0.1 \mathrm{~mol} / \mathrm{dm}^{3}\right.$ $\mathrm{KNO}_{3}$ ) was purged with argon for at least 5 min prior to and during the measurements. A $150 \mathrm{~W}$ xenon lamp (XBO-150), equipped with a water cooled housing and an automatically controlled monochromator (Instytut Fotonowy), was used as the light source. The working electrodes were irradiated in the range of 330-600 nm from the backside through the ITO layer in order to minimize the influence of the film thickness on the measured photocurrents. The measurements were controlled with the electrochemical analyzer Autolab, PGSTAT302N. The values of photocurrents were calculated using a custom-made software.

\subsection{Surface coverage tests}

Surface coverage tests of the studied photocatalysts were performed as follows. $20 \mathrm{mg}$ of the tested material were suspended in $2 \mathrm{~cm}^{3}$ of catechol solution in methanol $\left(1 \mathrm{mmol} / \mathrm{dm}^{3}\right)$. The mixture was sonicated for $5 \mathrm{~min}$ and centrifuged. Then the concentration of catechol in collected supernatant was determined by measuring the absorbance at $\lambda=280 \mathrm{~nm}$ (UV-vis 8453 Diode Array, Hewlett-Packard).

\subsection{Measurements of photocatalytic activity}

The photoactivity of modified photocatalysts were tested by measuring concentration of hydroxyterephthalic acid (TAOH) formed in the photoxidation reaction of terephthalic acid (TA). $12.4 \mathrm{mg}$ of the tested photocatalyst were sonicated for $30 \mathrm{~s}$ in $10 \mathrm{~cm}^{3}$ of water, then $10 \mathrm{~cm}^{3}$ of TA solution $\left(6 \cdot 10^{-4} \mathrm{~mol} / \mathrm{dm}^{3} \mathrm{TA}\right.$, $0.02 \mathrm{~mol} / \mathrm{dm}^{3} \mathrm{NaOH}, \mathrm{pH}=11$ ) was added. The suspension was placed in a quartz cuvette $\left(5 \mathrm{~cm}\right.$ dia., $1 \mathrm{~cm}$ optical path, $17 \mathrm{~cm}^{3}$ volume) and irradiated with the $150 \mathrm{~W}$ xenon lamp (XBO-150) equipped with a NIR filter $\left(0.1 \mathrm{~mol} / \mathrm{dm}^{3} \mathrm{CuSO}_{4}\right.$ solution in water) and $420 \mathrm{~nm}$ cut-off filter, or $320 \mathrm{~nm}$ cut-off filter. Tested samples were aerated and stirred during the measurement. The samples were collected after $0,15,30,60,120$, and 180 minutes (420 nm cut-off filter) or $0,5,10,15,20,25$ and 30 minutes ( 320 $\mathrm{nm}$ cut-off filter) of irradiation, filtered through CME syringe filters with $0.22 \mu \mathrm{m}$ pore size and subjected to the further analysis. The concentration of photogenerated TAOH was calculated from emission spectra measurements $\left(\lambda_{\mathrm{ex}}=315 \mathrm{~nm}\right.$, $\lambda_{\mathrm{em}}=426 \mathrm{~nm}$; PerkinElmer LS 55 Fluorescence Spectrometer). In final calculations a pseudo-zeroth-order kinetics was assumed based on the excess of TA. TAOH formation rates, calculated from the corresponding linear fits (Figure S1), were used to compare the efficiencies of $\mathrm{HO}^{\bullet}$ generation.

\subsection{Photodegradation of herbicides}

The photoactivity of the materials against model herbicides 2,4-dichlorophenoxyacetic acid (2,4-D) and 2,4,5-trichlorophenoxyacetic acid $(2,4,5-T)$ was tested in a quartz cuvette ( $5 \mathrm{~cm}$ dia., $1 \mathrm{~cm}$ optical path, $17 \mathrm{ml}$ volume). The herbicide and photocatalyst concentrations amounted $0.25 \mathrm{mM}$ and $1 \mathrm{~g} / \mathrm{dm}^{3}$, respectively. Irradiation was accomplished with the $150 \mathrm{~W}$ xenon lamp (XBO-150) equipped with a NIR filter $\left(0.1 \mathrm{~mol} / \mathrm{dm}^{3} \mathrm{CuSO}_{4}\right.$ solution in water) and $320 \mathrm{~nm}$ cut-off filter. The samples were aerated during the measurement. Each suspension was irradiated for $30 \mathrm{~min}$ and samples were collected every $5 \mathrm{~min}$. The changes in herbicide concentrations were calculated basing on the absorbance changes at 230 and $235 \mathrm{~nm}$ for 2,4-D and 2,4,5-T, respectively (UV-vis 8453 Diode Array, Hewlett-Packard).

\subsection{Water reduction tests}

The photoactivity of water reduction to hydrogen was tested in the following experiment. $10 \mathrm{mg}$ of the tested photocatalyst were suspended in $10 \mathrm{~cm}^{3}$ of water/methanol 1:1 (vol.) mixture. The suspension was placed in a quartz cuvette ( $5 \mathrm{~cm}$ dia., $1 \mathrm{~cm}$ optical path, $17 \mathrm{ml}$ volume) and irradiated for $2.5 \mathrm{~h}$ with the $150 \mathrm{~W}$ xenon lamp (XBO-150) equipped with a NIR filter $\left(0.1 \mathrm{~mol} / \mathrm{dm}^{3} \mathrm{CuSO}_{4}\right.$ solution in water) and $320 \mathrm{~nm}$ cut-off filter. The tested sample was stirred during the measurement and the samples (each one $50 \mu \mathrm{L}$ ) were collected every $25 \mathrm{~min}$. Generated hydrogen was determined on a gas chromatograph (Thermo Trace 1300) equipped with a FID detector and Carboxen 1010 PLOT column. Hydrogen formation rate constants were not calculated because of a complex kinetics (Figure S2a,b).

\section{Results and discussion}

\subsection{Structural, morphological and spectral properties}

The effect of surface modifications on photocatalytic activity was studied on two commercially available titanium dioxide samples: Hombikat UV100 and Evonik P25. The UV100 material is a nanocrystalline anatase (67\%, particle size ca. $9 \mathrm{~nm}$ ) forming aggregates with an average size of $1 \mu \mathrm{m}, 32$ while P25 is a highly active photocatalytic mixture of nanocrystalline (particle size ca. $25 \mathrm{~nm}$ ) anatase and rutile (78:14). ${ }^{33}$ Both materials also contain an amorphous phase. A small amount of $\mathrm{TiO}_{2}$ was grown on the surface of the powder materials by ALD using 10, $30,50,100$, and 200 growth cycles at a low deposition temperature of $150^{\circ} \mathrm{C}$. The average growth rate was about 0.5 $\AA$ Å/cycle for thicker reference $\mathrm{TiO}_{2}$ film sample deposited on a planar support. This value can vary from 0.15 to $0.6 \AA$ depending on the applied conditions. ${ }^{28}$ The layer growth starts with the formation of island-type crystalline particles ${ }^{25}$ which form a complete layer of $\mathrm{TiO}_{2}$ when at least 100-200 ALD cycles are applied. ${ }^{34}, 35$ The morphology of the synthesized surface depends on the deposition temperature and on the used support. At $150^{\circ} \mathrm{C} \mathrm{TiO}_{2}$ forms amorphous films with a smooth surface. ALD synthesis at higher temperatures results in the increase of the surface roughness and the particle size. ${ }^{36}$ This description is valid for films grown at planar supports, however, the situation for porous, powder supports may vary. Due to the developed surface of the coated powders a compact layer of amorphous $\mathrm{TiO}_{2}$ may not be formed even when 100-200 ALD cycles are applied. 
In both series of materials, XRD measurements show neither changes in phase composition nor in crystal size (Figure S3). Therefore, the influence of deposition conditions on the substrate was not observed. XRD revealed no new signals attributed to deposited titanium dioxide. Aarik et al. used the reflection high-energy electron diffraction to analyze $\mathrm{TiO}_{2}$ layers deposited by ALD and reported that the layer with a thickness of $2 \mathrm{~nm}$ has the structure of anatase. ${ }^{37}$ The phase transition is possible during the annealing process and the resulting polymorphic form of $\mathrm{TiO}_{2}$ depends on many factors, including calcination temperature, used precursors and thickness of the synthesized coat. ${ }^{25}$

P25 samples are less aggregated than Hombikat UV100 materials, as confirmed by the scanning electron microscopy (Figure 1). The effects of ALD modification were neither observed for P25 (Figure 1a,c), nor for UV100 based materials (Figure $1 \mathrm{~b}, \mathrm{~d}$ ). Specific surface area measurements, determined by the BET method (Table S1), revealed that for materials based on P25 the differences are minor (up to $2 \mathrm{~m}^{2} / \mathrm{g}$ ), however, in the case of UV100, more pronounced changes were observed. The biggest drop of the specific surface area caused by ALD modification was observed for 100@UV100 material (from 321.7 to $280.6 \mathrm{~m}^{2} / \mathrm{g}$ ). Also some fluctuations in the size and volume of pores were noticed for both series of materials.

TEM images were recorded for two materials with the most different specific surface area: UV100 and 100@UV100 (Figure 2 ). It appears that changes are very subtle and there is no plausible difference between the two measured samples. Moreover, it is hard to indicate the deposited $\mathrm{TiO}_{2}$ on the recorded images. There might be two reasons for this. The first one is related to the amount of deposited $\mathrm{TiO}_{2}$, too small to be detected. After 100 cycles of ALD the layer from 1.5 up to $6 \mathrm{~nm}$

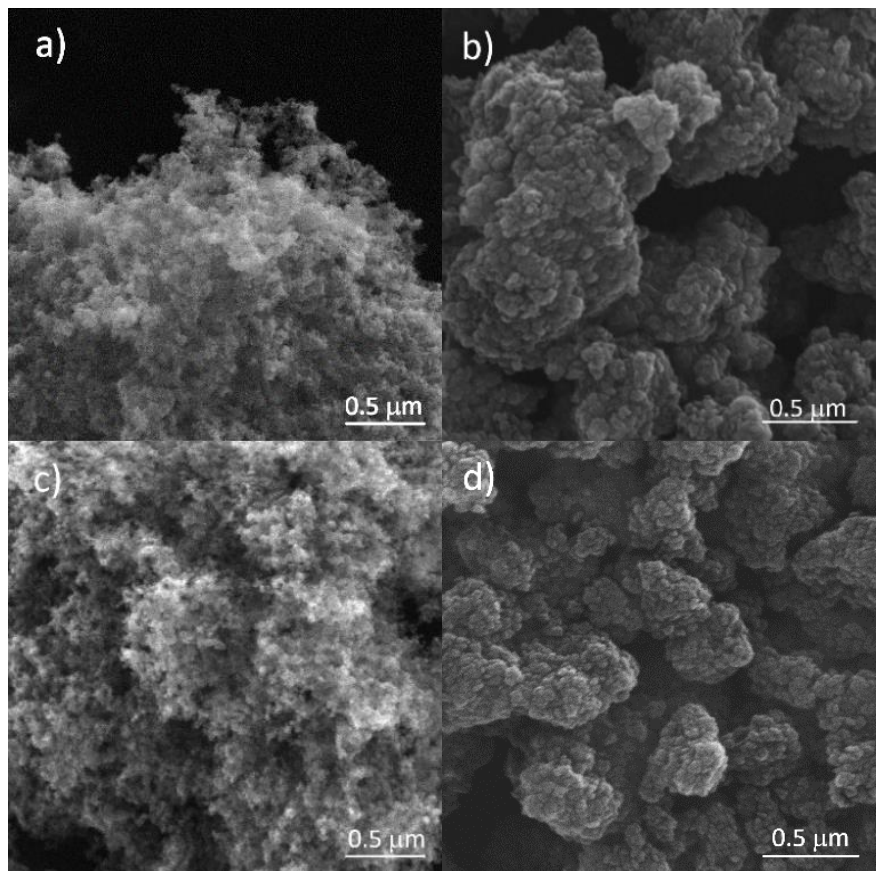

Fig. 1. SEM images of the materials before $(a, b)$ and after 200 cycles of $\operatorname{ALD}(c, d)$. The left and right columns present bare P25 $(a, c)$ and UV100 (b, d), respectively. can be expected on a flat surface. ${ }^{25}$ For powder $\mathrm{TiO}_{2}$ the deposited material may form a very inhomogeneous coverage, difficult to be recognized in the TEM picture. Formation of the amorphous phase, instead of a crystalline one, hard for observation in TEM pictures, is the second reason.

It is clear that the mode of $\mathrm{TiO}_{2}$ deposition on both materials is different. In the case of P25 the deposition of titania on single crystals (or grains composed of several crystals) slightly increasing their volume is plausible. These changes may be hardly noticeable on SEM images and should not influence significantly the specific surface area. In the case of UV100, deposited $\mathrm{TiO}_{2}$ probably covers large aggregates, reducing their surface area.

The new $\mathrm{TiO}_{2}$ layers may not only reduce specific surface area, but also influences the adsorption properties of the photocatalyst. Such effect was confirmed by the catechol adsorption tests (see Figure S4). It turned out that for P25-based materials the differences were insignificant and samples bound catechol similarly or slightly better than starting P25. In the case of UV100 the changes were more pronounced. After modification materials could bind more catechol despite a significant decrease of the specific surface area.

The surface properties after ALD modification were examined by X-ray photoelectron spectroscopy (XPS). Similarly to TEM measurements, two samples with the largest difference in specific surface area (UV100 and 100@UV100) were selected for comparative analysis. XPS, due to its relatively small sampling depth (ca. $6 \mathrm{~nm}$ ), ${ }^{38}$ allows observation of changes at the surface and in the near bulk of the sample. Fig. 3 shows the $\mathrm{Ti} 2 p$ and the $O 1 \mathrm{~s}$ regions of XPS spectra of the tested materials. Figures $3 a$ and $c$ show deconvoluted doublet assigned to the $p_{3 / 2}$ and $\mathrm{p}_{1 / 2}$ spin-orbit components of $\mathrm{Ti}^{4+}$, with no noticeable contribution of $\mathrm{Ti}^{3+}$ species. The $\mathrm{O} 1 \mathrm{~s}$ component of the XPS spectra (Fig. 3b, d) were deconvoluted into two Gaussian parts:

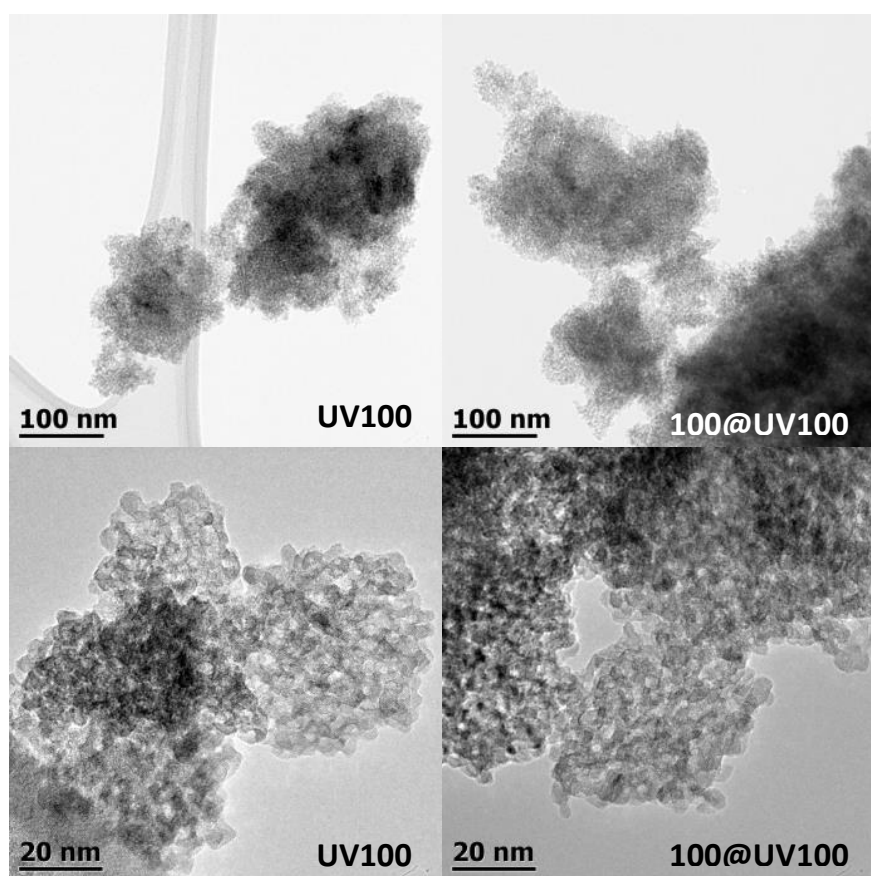

Fig. 2. TEM images of the UV100 and 100@UV100 materials. 

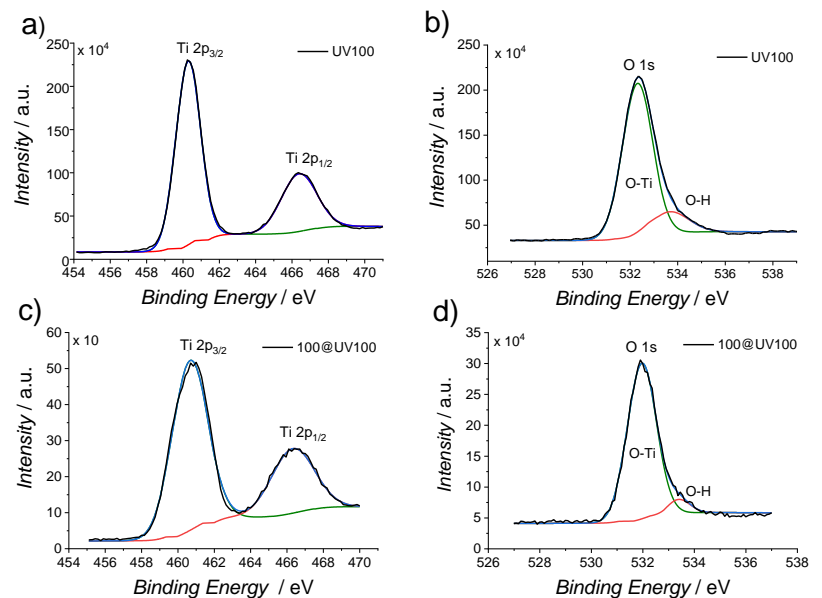

Fig. 3. XPS spectra: Ti $2 p(a, c)$ and $O 1 s(b, d)$ components recorded for $\mathrm{TiO}_{2} \mathrm{UV} 100(\mathrm{a}, \mathrm{b})$ and $100 @ \mathrm{UV} 100(c, d)$.

a low binding energy peak attributed to $\mathrm{O}^{2-}$ ions at intrinsic sites and a high binding energy peak attributed to non-lattice oxygen/surface -OH groups. ${ }^{39}$ The ratio between two components of the oxygen signal changes slightly for a material with an additional $\mathrm{TiO}_{2}$ layer. Nevertheless, the observed changes are negligible and therefore the spectra can be considered as identical within the experimental error.

For all ALD-synthesized materials diffuse reflectance spectra were also recorded. In the case of P25-based materials (Figure S5a) no significant changes in KM function were recorded, hence there was neither shift of the bandgap nor new band was formed. For modified UV100 samples (Figure S5b) some noticeable differences were observed for two materials (10@UV100 and 30@UV100) and a minor change for the 200@UV100 material. These materials exhibit a new small band with the maximum at $350 \mathrm{~nm}$.

\subsection{Spectroelectrochemistry - density of states}

Above mentioned experiments indicate that $\mathrm{TiO}_{2}$ deposition on bare P25 using ALD almost did not change structural, morphological and spectroscopic properties. Thus it can be concluded that at first glance these materials are almost identical to the original P25 sample. Nevertheless, Nitta et al. showed that apart from the examined parameters, the density of electronic states is of a great importance for the activity of the photocatalyst. ${ }^{30}, 40$ The applied modifications, although slightly affecting the sample, could have had an effect on the concentrations and distribution of surface defects. We have recently developed a method that allows determining the density of electron states for powder materials. ${ }^{31}$

Spectroelectrochemical measurements (SE-DRS) proved to be a method that is particularly sensitive to surface states. The results of SE-DRS measurements for all ALD-transformed materials are shown in Figure 4. Separated spectra for each sample are shown in the Figure $\mathrm{S6}$.

The presence of additional $\mathrm{TiO}_{2}$ deposits at bare titanium(IV) oxide has a noticeable effect on the DOS for both series of tested samples, although there are clear differences for each
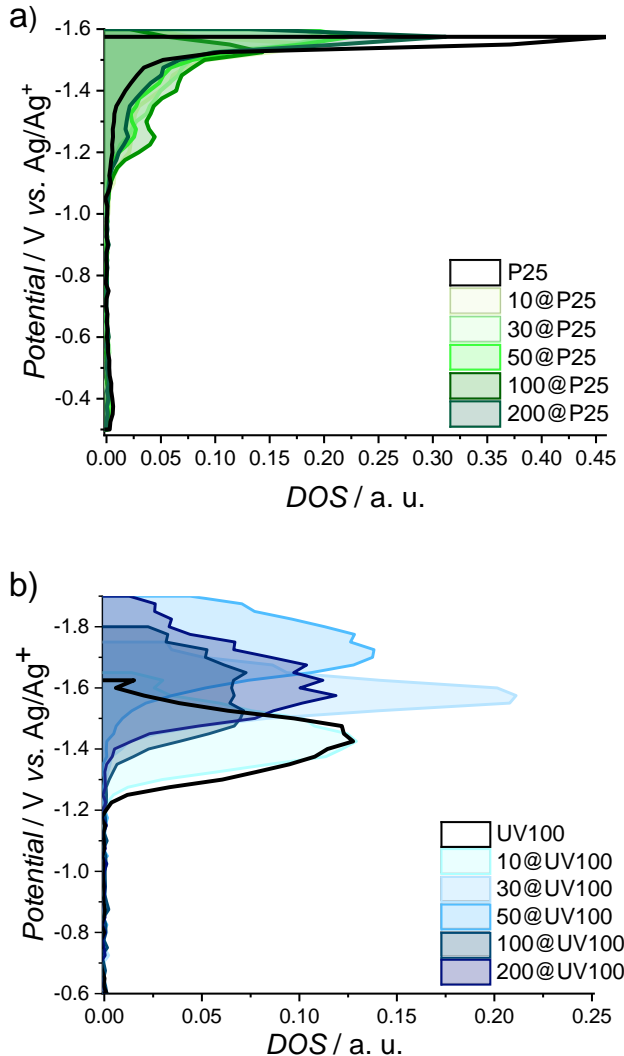

Fig. 4. Representative density of states patterns for $\mathrm{P} 25$ (a) and UV100 (b) after ALD application, measured in $0.1 \mathrm{~mol} / \mathrm{dm}^{3}$ $\mathrm{LiClO}_{4}$ acetonitrile electrolyte. The measurements were carried out for each working electrode under the same conditions in the inert atmosphere.

group of materials. In general, for ALD@UV100-based photocatalysts (Figure 4b) the density of states is shifted towards lower potentials compared to bare UV100. The atomic layer deposition seems to remove some states at higher potentials (in the range from $\mathrm{ca} .-1.2$ to $-1.3 \mathrm{~V} \mathrm{vs}$. $\mathrm{Ag} / \mathrm{Ag}^{+}$), causing enhancement of the reductive properties. In the case of ALD@P25 (Figure 4a) series the changes are more quantitative than qualitative - there is an increase of the density of states at lower energy (in the range from ca. -1.1 to $-1.5 \mathrm{~V} v$ s. $\mathrm{Ag} / \mathrm{Ag}^{+}$). For these materials the changes of electronic structures appear to be much smaller when compared to ALD@UV100 series. It is worth noting that the SE-DRS measurements in the case of $\mathrm{TiO}_{2}$ experiments provide information more about the distribution of the defects, including surface states, and to a lesser extent to free electrons in $\mathrm{CB}$, since the measurements encompass absorption changes in visible light range. ${ }^{41}$ Therefore more significant qualitative changes in DOS observed for ALD@UV100 than for ALD@P25 series are in accordance with more pronounced changes of the surface of UV100 when modified with ALD (compare BET and catechol adsorption tests).

The presence of additional $\mathrm{TiO}_{2}$ deposits at bare titanium(IV) oxide has a noticeable effect on the DOS for both series of tested samples, although there are clear differences for each group of materials. In general, for ALD@UV100-based 
photocatalysts (Figure 4b) the density of states is shifted towards lower potentials compared to bare UV100. The atomic layer deposition seems to remove some states at higher potentials (in the range from $\mathrm{ca} .-1.2$ to $-1.3 \mathrm{~V} v \mathrm{vs}$. $\mathrm{Ag} / \mathrm{Ag}^{+}$), causing enhancement of the reductive properties. In the case of ALD@P25 (Figure 3a) series the changes are more quantitative than qualitative - there is an increase of the density of states at lower energy (in the range from ca. -1.1 to $-1.5 \mathrm{~V} v \mathrm{vs}$. $\mathrm{Ag} / \mathrm{Ag}^{+}$). For these materials the changes of electronic structures appear to be much smaller when compared to ALD@UV100 series. It is worth noting that the SE-DRS measurements in the case of $\mathrm{TiO}_{2}$ experiments provide information more about the distribution of the defects, including surface states, and to a lesser extent to free electrons in $\mathrm{CB}$, since the measurements encompass absorption changes in visible light range. ${ }^{41}$ Therefore more significant qualitative changes in DOS observed for ALD@UV100 than for ALD@P25 series are in accordance with more pronounced changes of the surface of UV100 when modified with ALD (compare BET and catechol adsorption tests).

\subsection{Photoelectrochemistry}

It is possible that the modification which influences DOS will probably also affect other properties of photo-excited materials. Measurements of photocurrents (Figure 5) seem to confirm these assumptions. On the one hand, obtained results do not show changes in the spectral range of the photoelectrochemical activity of the tested samples, but on the other hand, there is a significant decrease in photon to current conversion efficiency between bare P25 and ALD@P25 samples. Deterioration of anodic photocurrents for these materials can be associated with decreasing efficiency of interfacial electron transfer taking place at studied electrodes. For ALD@UV100 samples the changes were also observed, although they exhibited a different behaviour and were much smaller.

The influence of oxygen or carbon dioxide on photocurrents was also investigated. For titanium dioxide the anodic photocurrents are observed within a wide range of potentials, nevertheless, at negative potentials cathodic photocurrents can appear in the presence of an electron photocurrents are observed within a wide range of potentials, nevertheless, at

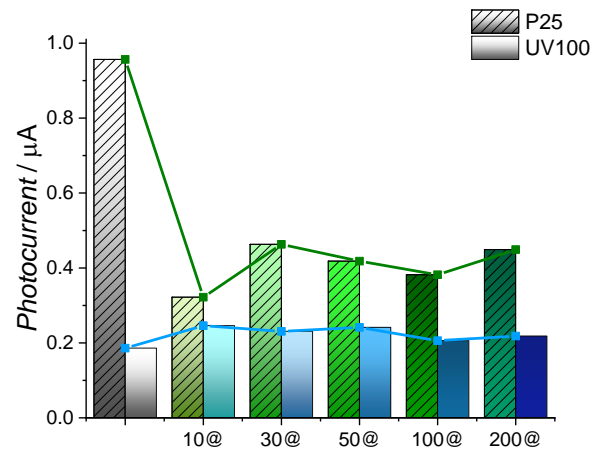

Fig. 5. Photocurrent generation for studied materials recorded at $1 \mathrm{~V}$ vs. $\mathrm{Ag} / \mathrm{AgCl}$, at $\lambda=340 \mathrm{~nm}$ in deoxygenated $0.1 \mathrm{~mol} / \mathrm{dm}^{3}$ $\mathrm{KNO}_{3}$. negative potentials cathodic photocurrents can appear in the presence of an electron acceptor. A decrease of anodic photocurrents or even their switching to cathodic ones after introducing an electron acceptor (e.g., oxygen) confirms the material ability to reduction of the introduced species by photogenerated electrons. It must be noted that morphological properties of the electrode or changes of the electrolyte $\mathrm{pH}$ have a significant impact on the resulting photocurrent. ${ }^{42,} 43$ Therefore, a carbonate buffer was used as the electrolyte to minimize the effect of $\mathrm{pH}$ changes and all measurement (in the presence of oxygen, argon or $\mathrm{CO}_{2}$ ) were performed for the same electrode. The photocurrent generation ability was different for various materials. In order to correctly assess the influence of oxygen and $\mathrm{CO}_{2}$ on generated photocurrent for a series of ALD-modified materials, the normalized difference between photocurrent recorded in the presence of oxygen or carbon dioxide $\left(\left(I_{\mathrm{p}}\left(\mathrm{O}_{2}\right), I_{\mathrm{p}}\left(\mathrm{CO}_{2}\right)\right)\right.$ and in the electrolyte saturated with argon $\left(I_{p}(\mathrm{Ar})\right)$ was calculated (Figure 6$)$.

The sensitivity to oxygen and carbon dioxide for both series of the tested materials changed drastically after ALD modification. In the case of ALD@P25 materials almost none of them (except for 50@P25) shows any effect of $\mathrm{O}_{2}$ or $\mathrm{CO}_{2}$ and presence. The 50@P25 photocatalyst offers the highest density of electronic states at low energies, close to $-1.2 \mathrm{~V} v \mathrm{v}$. $\mathrm{Ag} / \mathrm{Ag}^{+}$. In this case of the ALD@UV100 series only 100@UV100

a)

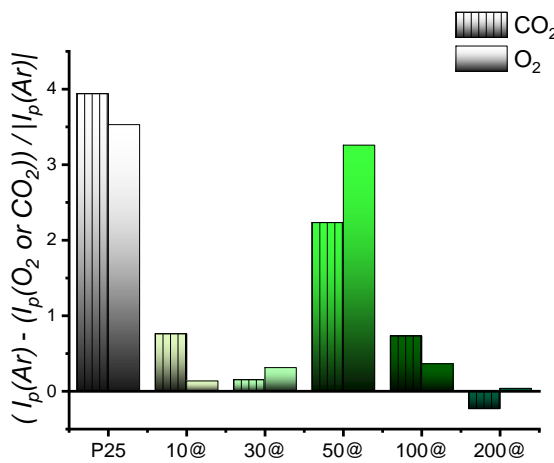

b)

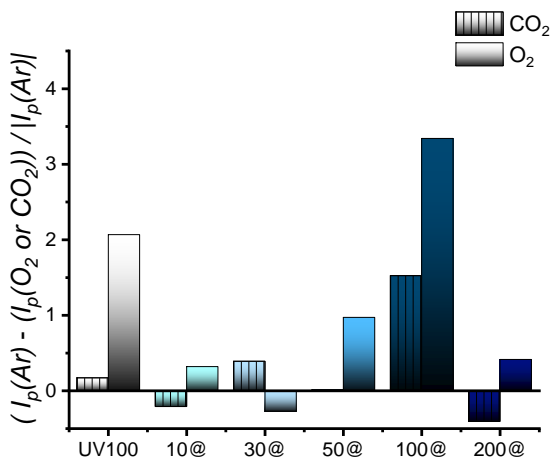

Fig. 6. Normalized differences between photocurrents recorded in the presence of oxygen or carbon dioxide $\left(\left(I_{\mathrm{p}}\left(\mathrm{O}_{2}\right), I_{\mathrm{p}}\left(\mathrm{CO}_{2}\right)\right)\right.$ and in the electrolyte saturated with argon $\left(I_{\mathrm{p}}(\mathrm{Ar})\right)$ for ALD@P25 (a) ALD@UV100 (b) materials. The values were recorded at the potential of $-0.2 \mathrm{~V} v \mathrm{~s}$. $\mathrm{Ag} / \mathrm{AgCl}$ at $\lambda=340 \mathrm{~nm}$. 
appeared sensitive to $\mathrm{O}_{2}$ and $\mathrm{CO}_{2}$. This material shows even higher ability to reduce the gases than bare UV100. Although its conduction band edge appears at lower potentials compared to 10@UV100 and UV100, this material should not offer the best reducing properties as could be concluded from SE-DRS measurements (e.g., 50@UV100 should offer a higher energy of electrons in the conduction band). Interestingly, 100@UV100 reduces photocatalytically $\mathrm{O}_{2}$ and $\mathrm{CO}_{2}$ most efficiently despite of the smallest specific surface area (Table S1).

\subsection{Photocatalytic activity}

The changes in photocatalytic activity were also studied by measuring the ability of hydrogen production from water for both series of materials (Figure 7). Experiments were performed in the water-methanol solution under inert atmosphere to prevent a competitive reaction of oxygen reduction. Under these conditions the efficiency of hydrogen production should depend only on the reduction abilities of the tested sample. Addition of methanol was necessary to quench photogenerated holes and to enable studies of only the reduction counterpart of the reaction.

The hydrogen generation of tested materials has a complex kinetics. In the first phase the reaction is slower, then it accelerates and the rate stabilizes (Figure S2). Such kinetics could be caused, inter alia, by sorption of photogenerated hydrogen in the photocatalyst structure. A significantly larger pores volume in the case of UV100 materials compared to P25 ones (Table S1) is in accordance with a larger effect of the apparent reaction acceleration observed for the UV100 series. A deterioration of photocatalytic activity of P25 when modified by ALD and relatively small influence of ALD modifications on photoreactivity of UV100 (Figure 7) follow the effect of the modifications on photocurrent generation (Figure 5). These results demonstrate that the described apparently insignificant The process of photoconversion of terephthalic acid (TA) to hydroxyterephthalic acid (TAOH) was used to compare the efficiencies of hydroxyl radicals generation upon UV irradiation. modifications, especially in the case of ALD@P25 materials, can influence the overall photocatalytic reduction activity.

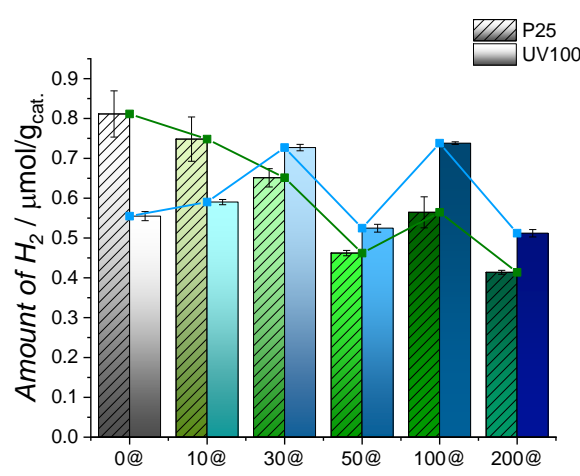

Fig. 7. Amounts of $\mathrm{H}_{2}$ photogenerated from water-methanol mixture (1:1) in the presence of modified materials after $2 \mathrm{~h}$ of irradiation $(\lambda>320 \mathrm{~nm})$. Presented results are calculated for $1 \mathrm{~g}$ of the photocatalyst used in concentration of $0.775 \mathrm{~g} / \mathrm{dm}^{3}$.
The rates of $\mathrm{TAOH}$ generation for each sample (Figure 8) were constant within the tested reaction time (30 $\mathrm{min})$, thus formation rates were calculated from the corresponding linear fits (see Figure S1) and used to compare the efficiencies of $\mathrm{HO}^{\circ}$ generation. In the case of P25-based samples, in the series 10@P25-100@P25 a decrease of photoactivity can be observed, in accordance with the trend described for hydrogen generation reaction. As reported previously, UV100-based materials show a significantly lower activity than P25 in this reaction. ${ }^{44}$ ALD modifications of UV100 typically decreased its photocatalytic activity. As far as in the case of ALD@P25 samples activity decrease correlates with previous results while the observed trend is surprising for the UV100-based materials.

More pronounced differences in photocatalytic activity of the tested materials can be observed in the case of degradation of herbicides, 2,4,5-trichlorophenoxyacetic acid (2,4,5-T) and 2,4-dichlorophenoxyacetic acid (2,4-D) (Figure 9). The photocatalysts activity profiles for these reactions show the same features, both for 2,4-D and 2,3,4-T. This probably results from a similar chemical structures of 2,4-D and 2,3,4-T and mechanism of their degradation, involving mainly hydroxyl radicals and holes. ${ }^{45,} 46$ Presented results are another example of noticeable changes in photocatalytic activity caused by minor modification in the surface structure.

Until now the photoactivity of materials irradiated with ultraviolet light $\left(h v>E_{\mathrm{g}}\right)$ was discussed. Titania modifications may influence the electronic structure of the samples and can have an impact on photoactivity, including spectral range of utilized light (photon energy higher or lower than $E_{\mathrm{g}}$ ). In particular, electronic states localized within the band gap drive the primary photocatalytic processes. Their presence has a rather minor influence on light absorption, as the Urbach tail absorbance is much weaker than the absorbance related to the interband electron transfer. ${ }^{18,}{ }^{47}$ However, ES may trap charges and mediate interfacial redox reactions. When the photon energy is smaller than $E_{g}$, electronic states play a pivotal role in excitation of the semiconductor and induction of a photocatalytic reaction. Thus it can be expected that changes in

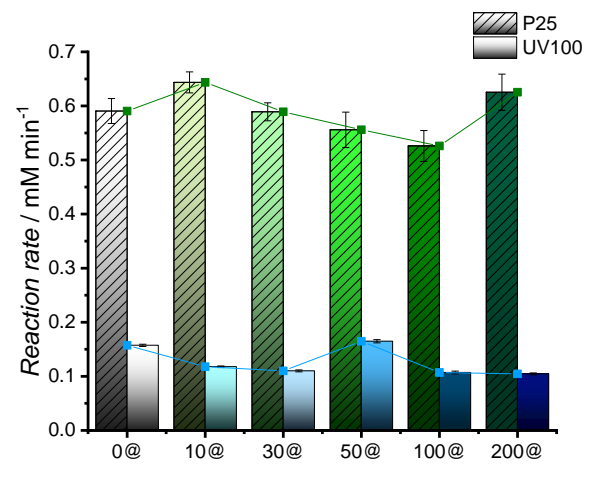

Fig. 8. Reaction rates of $\mathrm{TAOH}$ formation in the process of photocatalytic oxidation of TA in the presence of tested materials under UV irradiation $(\lambda>320 \mathrm{~nm})$. Presented results are calculated for $1 \mathrm{~g}$ of the photocatalyst used in concentration of $0.775 \mathrm{~g} / \mathrm{dm}^{3}$. 
ES distribution may alter the photoactivity. To demonstrate this effect, we checked the TAOH generation rates (Figure 10) upon visible light irradiation $(\lambda>420 \mathrm{~nm})$.

The observed influence of ALD modification is completely different from that described above for samples irradiated with UV light $(\lambda>320 \mathrm{~nm})$. The reaction rates of $\mathrm{TAOH}$ production are about two times (or even more) higher for 10@P25, 30@P25, 10@UV100 and 30@UV100 compared to corresponding bare titanium(IV) oxides. This effect is probably combined with various roles (charge traps, electron transfer mediators, chromophoric centers) of electronic states localized within the band gap, changes in surface chemistry (distribution of defects) or the influence of changes in amorphous phase deposits. Obtained results also indicate, that modification of electronic structure may have significant impact on photosensitization of $\mathrm{TiO}_{2}$ materials. For better understanding of this effect, experiments on TAOH photogeneration upon the visible light irradiation $(\lambda>420 \mathrm{~nm}$ ) have been done for cat@P25, 10@P25, 30@P25 and bare P25 (Figure 11). In this series, catechol adsorbed at P25 (cat@P25) is a well-recognized photosensitized $\mathrm{TiO}_{2}$-based system. ${ }^{48-50}$ At the beginning 10@P25 and 30@P25 are less active than cat@P25, which appears very active within the first $20 \mathrm{~min}$ but then deactivates due to irreversible photooxidation of catechol. However, after $2 \mathrm{~h}$ these differences start to blur and mentioned materials can even surpass the activity of the photosensitized $\mathrm{TiO}_{2}$.
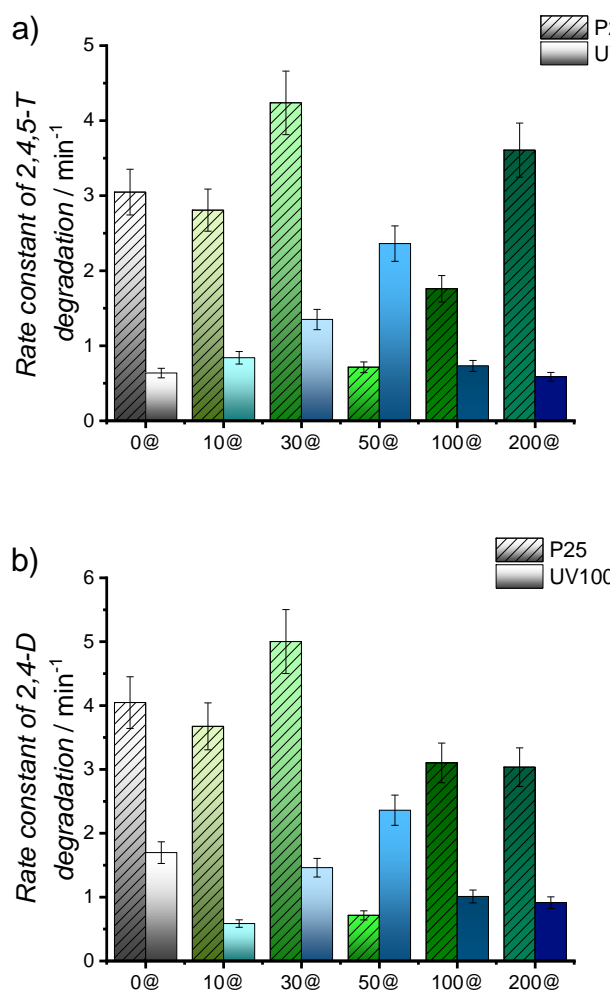

Fig. 9. Rate constants of 2,4,5-T (a) and 2,4-D (b) herbicides degradation in the presence of tested materials under light irradiation $(\lambda>320 \mathrm{~nm})$.
Interestingly, minor changes in the surface structure caused a relatively large change in activity, when compared to P25. At the first glance, there is no reason to assume that modifications caused by the deposition of small amounts of $\mathrm{TiO}_{2}$ can be considered as an extremely intrusive modification method, due to mild reaction conditions (compare Experimental part). In fact, any surface modification method can affect the photocatalytic properties of a semiconductor. For example, some physicochemical changes caused only by mechanical treatment (grinding) of $\mathrm{TiO}_{2}$ can significantly decrease its photocatalytic activity. ${ }^{51}$

\subsection{Influence of synthesis conditions}

Usually the influence of synthesis conditions on the resulting activity of photocatalysts is not taken into account, but, as mentioned above, even insignificant modifications can alter photocatalytic properties. Therefore, we analyzed how protocols for typical procedures of gold nanoparticles deposition themselves can influence the overall photoactivity of P25 and UV100. Three different protocols were used: GNps

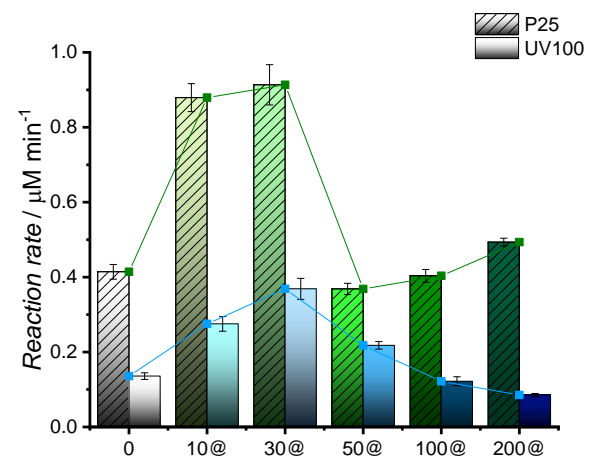

Fig. 10. Reaction rates of $\mathrm{TAOH}$ formation in the process of photocatalytic oxidation of TA in the presence of tested materials under visible light irradiation $(\lambda>420 \mathrm{~nm})$. Presented results are calculated for $1 \mathrm{~g}$ of the photocatalyst used in concentration of $0.775 \mathrm{~g} / \mathrm{dm}^{3}$.

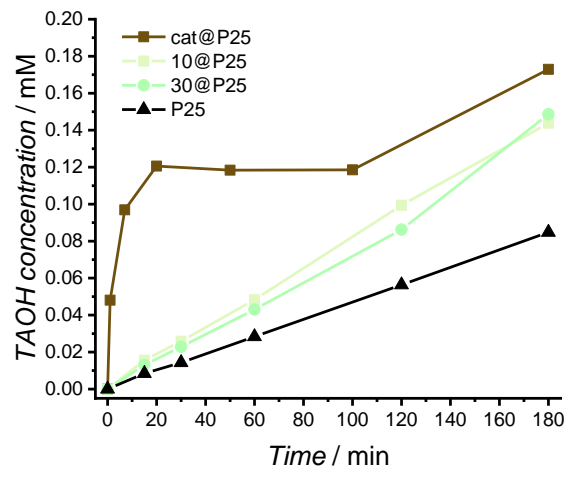

Fig. 11. TAOH formation in the process of photocatalytic oxidation of TA in the presence of tested materials after $180 \mathrm{~min}$ of irradiation $(\lambda>420 \mathrm{~nm})$. Presented results are calculated for $1 \mathrm{~g}$ of the photocatalyst used in concentration of $0.775 \mathrm{~g} / \mathrm{dm}^{3}$. 
photodeposition, $\mathrm{AuCl}_{3}$ reduction with sodium borohydride and $\mathrm{AuCl}_{3}$ reduction with sodium citrate and temperature. Synthesis of all series of materials were performed with and without gold precursor, yielding twelve materials. In all cases the reducing agent was used in a large excess.

For the synthesized gold-containing samples the absorption band with maximum at ca. $550 \mathrm{~nm}$ was observed (Figure S7), which can be attributed to the surface plasmonic resonance of spherical gold nanoparticles. ${ }^{52,53}$ Also the presence of XRD signals characteristic for $\mathrm{Au}$ ( $2 \theta$ values of $44.4^{\circ}, 64.5^{\circ}$ and $77.5^{\circ}$ ) confirmed the presence of this metal nanoparticles at the surface of the photocatalysts (Figure S8). ${ }^{54}$ A more detailed characterization of the GNps distribution at the titania surface was performed using EDS measurement (Figure S9). It appears that in all synthesized samples the deposited gold is spread evenly. EDS, DRS and XRD results differ for various synthesis methods involving P25 and UV100 materials. It can be concluded be concluded that the size and shape of gold nanoparticles are Determined by the protocols and the used support. The dependence of the size of GNps on the size of $\mathrm{TiO}_{2}$ particles in the case of photodeposition synthesis was discussed by Kowalska et al..$^{55}$ They correlated this dependence with the concentration of surface defects that can serve as nucleation centers. Materials with the highest density of defects at fine titania particles enabled formation of the largest GNps. ${ }^{56} \mathrm{~A}$ similar effect may be responsible for the differences in $\mathrm{Au}$ nanoparticles deposited at P25 and UV100 in the case of all applied synthetic methods. For materials obtained in the absence of gold precursor DRS spectra and XRD measurements were identical within P25 and UV100 series (Figures S10, S11).

Surprisingly, spectroelectrochemical measurements of _(xx)@P25 and _(xx)@UV100 materials (without GNps) revealed even more pronounced changes in the density of states (Figure 12 - separated spectra for each sample are shown in the Figure S12) compared to previously discussed ALDmodified photocatalysts (Figure 4). In the P25 series the changes are more related to the increase of the density of states in the range from -1.1 to $-1.4 \mathrm{~V} v s$. $\mathrm{Ag} / \mathrm{Ag}^{+}$. In contrast, for UV100-based materials, a decrease in the density of lower energy states (at -1.2 to $-1.4 \mathrm{~V}$ ) can be observed. Similar trends of DOS changes were observed also for ALD-modified materials: an amplification and vanishing of low energy electronic states for P25 and UV100 series, respectively.

Also in this case the surface ability to bind catechol was examined. Both series, with and without GNps, exhibit different binding properties (Figure S13). For most samples the amount of bound 1,2-dihydroxybenzene decreases or remains almost unchanged after application of the synthesis protocols. Analysis of Figure S9 shows that presence of GNps plays a secondary role in catechol adsorption, while a pivotal role can be attributed to the application of particular reduction conditions. $\mathrm{Au}\left(\mathrm{NaBH}_{4}\right) @ \mathrm{P} 25$ is the only exception, since clearly lower amounts of catechol are adsorbed at this sample compared to _ $\left(\mathrm{NaBH}_{4}\right) @ \mathrm{P} 25$. TAOH generation induced by visible light also followed the pseudo-zeroth-order kinetics. Figure 13 presents reaction rates of $\mathrm{HO}^{\cdot}$ generation. $\mathrm{Au}\left(\mathrm{NaBH}_{4}\right) @ \mathrm{P} 25$ turned out to be the most active material, showing 10 times higher activity than bare P25 under the same conditions. Accordingly, $\mathrm{Au}\left(\mathrm{NaBH}_{4}\right) @ U V 100$ appeared slightly more active than both starting UV100 and corresponding_( $\left(\mathrm{NaBH}_{4}\right) @ U V 100$. It can be concluded, that the synthesis involving reduction by sodium borohydride gives the most optimal gold nanoparticles. The remaining materials with GNps are comparable or less active than bare P25 and UV100. Some authors explain the decreasing photoactivity in oxidation reactions by recombination of charge carriers at gold nanoparticles. ${ }^{57}$

Treated materials without deposited gold show TAOH generation efficiencies similar or lower than those observed for the materials with deposited gold. The only exception is _(Photo)@UV100, for which the rate of TA hydroxylation is ca. 5 times higher compared to UV100 and ca. 6 times higher compared to Au(Photo)@UV100. This remarkable increase in photoactivity results only from the treatment by methanol and light, which has a more significant influence on photoactivity than deposited gold nanoparticles. This clearly demonstrates how significant changes in the activity of photocatalysts can be induced by a relatively simple and apparently innocent treatment. At the same time our results show how cautiously one should interpret the effects of surface modifications on the overall photocatalytic activity of wide bandgap semiconductors.
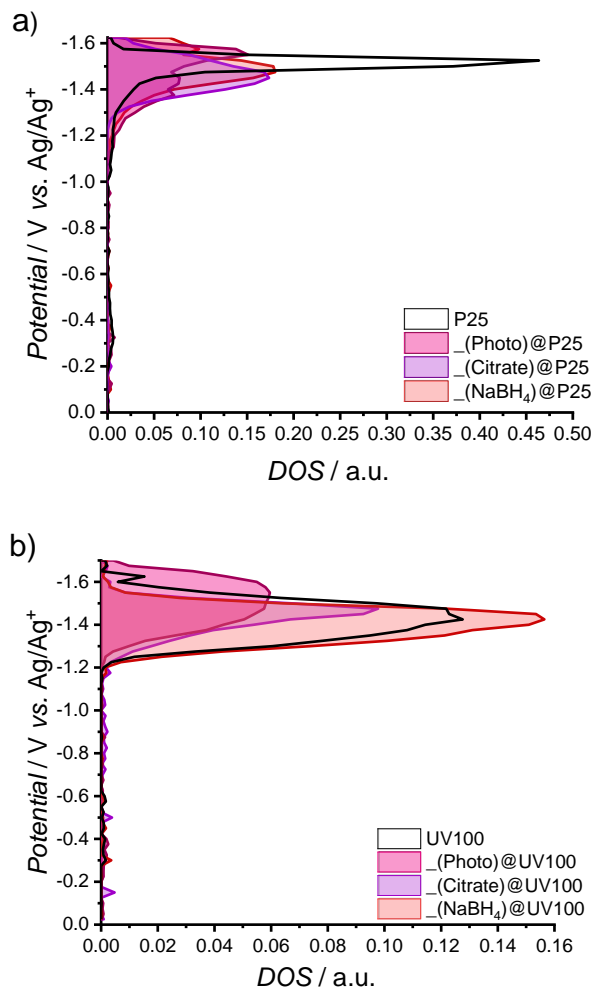

Fig. 12. Density of electronic states patterns for P25 (a) and UV100 (b) measured in $0.1 \mathrm{~mol} / \mathrm{dm}^{3} \quad \mathrm{LiClO}_{4}$ acetonitrile electrolyte. The measurements were carried out for each working electrode under the same conditions, in the inert atmosphere. 
a)
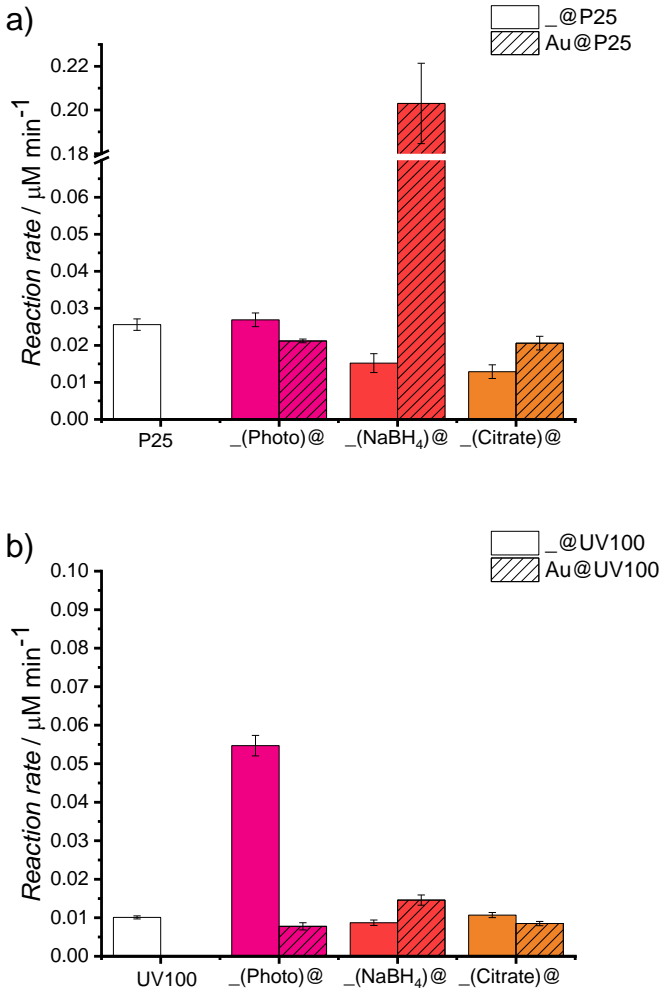

Fig. 13. Reaction rates of TAOH formation in the process of photocatalytic oxidation of TA in the presence of Au NP visible light irradiation $(\lambda>420 \mathrm{~nm})$. Presented results are calculated for $1 \mathrm{~g}$ of the photocatalyst used in concentration of 0.775 $\mathrm{g} / \mathrm{dm}^{3}$.

\subsection{Influence of insignificant modifications}

The results obtained for materials treated in the same way as those with gold have shown once again that even seemingly insignificant factors can noticeable affect the activity. The synthetic protocols (including ALD) probably did not affect the semiconductor bulk, but very likely changed the surface. The mentioned procedures may influence the content of the amorphous deposit. They could also affect chemistry of the crystal surface by changing the distribution of structural defects, such as oxygen vacancies or $\mathrm{OH}$ bridges. Each treatment could influence the surface of semiconductor in a different way making correlation of a particular handling with specific surface changes and the activity practically unforeseeable, although finding of this correlation would be very beneficial. Currently, both theoretical and experimental studies are aimed to shed some light on this problem. ${ }^{58-61}$

Every factor described in this work influencing the photocatalytic activity of the tested samples was already described in literature - to a certain extent - in respect to its influence on the photocatalyst performance. In the case of ALD, the photocatalytic properties of $\mathrm{TiO}_{2}$ layers synthesized on flat substrates (e.g., $\mathrm{Si}$, glass) were analyzed, applying from hundreds to thousands deposition cycles. For these relatively thick layers authors were able to reveal some trends in activity changes. ${ }^{27}, 62,63$ Studying the influence of conditions of nanoparticles deposition on the surface of semiconductors the main two factors can be considered: temperature and the reductants. A clear, foreseeable influence of temperature can be observed for relatively wide temperature ranges, containing materials based on P25 (a) and UV100 (b)) under influencing, for instance, the phase composition, crystal size, etc. ${ }^{64-66}$ In our case applied mild temperatures (up to 100 or $150^{\circ} \mathrm{C}$ in the case of the material boiling or ALD deposition, respectively) do not generate such obvious effects. Similarly, reducing agents are examined most frequently in a wide range of concentrations (usually large) and longer exposure times to acquire a generalized description of their impact. ${ }^{8,} 67,68$ In conclusion, all mentioned parameters which affect the photocatalytic properties of our samples were studied under conditions that definitely cannot be described as "insignificant". In those cases the authors could find some general trends, however, their extrapolation to our conditions (a few ALD cycles, low temperatures, low concentration of reductants, etc.) should indicate a negligible influence, whereas our results remain in contradiction with such conclusions. The situation is even more complex: in each considered system there is more than one seemingly insignificant factor that affects the results (e.g., temperature and number of ALD cycles). Only a significant increase of the contribution of one selected factor to the overall photocatalytic properties would enable a generalized description of its impact on the activity, but this would eliminate the possibility of studying the influence of other "seemingly irrelevant" parameters. The goal of our work was to show how apparently insignificant factors can significantly influence the photocatalytic activity of a semiconductor photocatalyst.

\section{Conclusions}

In this work we have shown that slight surface modifications of titanium dioxide ("insignificant" at the first glance), such as exposition to reducing or oxidizing agents and deposition of small amounts of the semiconductor material at the surface, may strongly affect the activity of studied materials. Although most of "classical" characterization methods (XRD, UV-vis, porosimetry, SEM, TEM, XPS) does not show any differences between original and treated samples, methods sensitive to surface changes, like spectroelectrochemical DOS analysis or catechol adsorption tests revealed a significant influence of such treatments on catalytic, photocatalytic and photoelectrochemical properties.

In the case of ALD modified samples, the reaction rates of TAOH production under visible light irradiation $(\lambda>420 \mathrm{~nm})$ were about two times (or even more) higher for 10@P25, 30@P25, 10@UV100 and 30@UV100 compared to corresponding bare titanium(IV) oxides. For materials obtained with protocols of gold nanoparticles deposition one sample, _(Photo)@UV100, exhibited the reaction rate of TA hydroxylation ca. 5 times higher compared to bare UV100 and ca. 6 times higher compared to Au(Photo)@UV100. This remarkable increase in photoactivity resulted only from the treatment by methanol and light, which have a more significant influence on photoactivity than deposited gold nanoparticles. 
We have demonstrated, that comparative studies on photoactivity of various semiconductors must take into account not only primary effects of fundamental, structural and elemental modifications, but also much more subtle, insignificant surface treatment, which may noticeably influence surface chemistry. We demonstrated that SE-DRS can be considered as a useful tool to study these effects.

\section{Conflicts of interest}

There are no conflicts to declare.

\section{Acknowledgements}

The work was supported by National Science Centre, Poland (NCN) within the project no. 2015/19/B/ST5/00950. KM acknowledges the support by the Foundation for Polish Science (FNP) within the TEAM project (POIR.04.04.00-00-3D74/16) project. This research was also supported by the Finnish Centre of Excellence in Atomic Layer Deposition.

\section{Notes and references}

1. A. Meng, L. Zhang, B. Cheng and J. Yu, Adv. Mater., 2019, 1807660.

2. M. Buchalska, M. Kobielusz, A. Matuszek, M. Pacia, S. Wojtyła and W. Macyk, ACS Catal., 2015, 5, 7424-7431.

3. S. G. Kumar and L. G. Devi, J. Phys. Chem. A, 2011, 115, 13211-13241.

4. M. Pelaez, N. T. Nolan, S. C. Pillai, M. K. Seery, P. Falaras, A. G. Kontos, P. S. Dunlop, J. W. J. Hamilton, J. A. Byrne and K. O'shea, Appl. Catal. B: Environ., 2012, 125, 331-349.

5. A. Šuligoj, I. Arčon, M. Mazaj, G. Dražić, D. Arčon, P. Cool, U. L. Štangar and N. N. Tušar, J. Mater. Chem. A, 2018, 6, 9882-9892.

6. M.-C. Wu, W.-K. Huang, T.-H. Lin and Y.-J. Lu, Appl. Surf. Sci., 2019, 469, 34-43.

7. Z. Tachan, I. Hod and A. Zaban, Adv. Energy Mater., 2014, 4, 1301249.

8. W. Fang, M. Xing and J. Zhang, J. Photochem. Photobiol. C: Photochem. Rev., 2017, 32, 21-39.

9. D. Jiang, Y. Xu, D. Wu and Y. Sun, Appl. Catal. B: Environ., 2009, 88, 165-172.

10. A. Bjelajac, V. Djokić, R. Petrović, N. Bundaleski, G. Socol, I. N. Mihailescu, Z. Rakočević and D. Janaćković, Ceram. Int., 2017, 43, 15040-15046.

11. D. A. Panayotov and J. R. Morris, Surf. Sci. Rep., 2016, 71, 77-271.

12. X. Ning, W. Zhen, Y. Wu and G. Lu, Appl. Catal. B: Environ. 2017, 226, 373-383.

13. R. Sánchez-de-Armas, J. Oviedo, M. Á. San Miguel and J. F. Sanz, J. Phys. Chem. C, 2011, 115, 11293-11301.

14. D. Jiang, Y. Xu, D. Wu and Y. Sun, J. Solid State Chem., 2008, 181, 593-602.

15. K. Qi, F. Zasada, W. Piskorz, P. Indyka, J. Gryboś, M Trochowski, M. Buchalska, M. Kobielusz, W. Macyk and Z. Sojka, J. Phys. Chem. C, 2016, 120, 5442-5456.
16. R. Asahi, T. Morikawa, T. Ohwaki, K. Aoki and Y. Taga, Science, 2001, 293, 269-271.

17. Á. Kmetykó, K. Mogyorósi, P. Pusztai, T. Radu, Z. Kónya, A. Dombi and K. Hernádi, Materials, 2014, 7, 7615-7633.

18. H. Kisch, Semiconductor photocatalysis: principles and applications, John Wiley \& Sons, 2014.

19. J. Jupille and G. Thornton, Defects at oxide surfaces, Springer, 2015.

20. J. Yan, G. Wu, N. Guan, L. Li, Z. Li and X. Cao, Phys. Chem. Chem. Phys., 2013, 15, 10978-10988.

21. B. Ohtani, Catalysts, 2016, 6, 192-197.

22. A. Yamakata, J. J. M. Vequizo and H. Matsunaga, J. Phys. Chem. C, 2015, 119, 24538-24545.

23. M. Kong, Y. Li, X. Chen, T. Tian, P. Fang, F. Zheng and X. Zhao, J. Am. Chem. Soc., 2011, 133, 16414-16417.

24. T. livonen, J. Hämäläinen, B. Marchand, K. Mizohata, M. Mattinen, G. Popov, J. Kim, R. A. Fischer and M. Leskelä, J. Vac. Sci. Technol., A, 2016, 34, 01A109.

25. J.-P. Niemelä, G. Marin and M. Karppinen, Semicond. Sci. Technol., 2017, 32.

26. M. Leskelä and M. Ritala, Thin Solid Films, 2002, 409, 138146.

27. M. Buchalska, M. Surówka, J. Hämäläinen, T. livonen, M. Leskelä and W. Macyk, Catal. Today, 2015, 252, 14-19.

28. M. Ritala, M. Leskelä, L. Niinistö and P. Haussalo, Chem. Mater., 1993, 5, 1174-1181.

29. J. Kimling, M. Maier, B. Okenve, V. Kotaidis, H. Ballot and A. Plech, J. Phys. Chem. B, 2006, 110, 15700-15707.

30. E. Świętek, K. Pilarczyk, J. Derdzińska, K. Szaciłowski and W. Macyk, Phys. Chem. Chem. Phys., 2013, 15, 14256-14261.

31. M. Kobielusz, K. Pilarczyk, E. Świętek, K. Szaciłowski and W. Macyk, Catal. Today, 2018, 309, 35-42.

32. H. Jensen, K. D. Joensen, J.-E. Jørgensen, J. S. Pedersen and G. Søgaard, J. Nanoparticle Res., 2004, 6, 519-526.

33. B. Ohtani, O. O. Prieto-Mahaney, D. Li and R. Abe, J. Photochem. Photobiol. A: Chem., 2010, 216, 179-182.

34. M. Ritala, M. Leskelä, L.-S. Johansson and L. Niinistö, Thin Solid Films, 1993, 228, 32-35.

35. N. Dolgushev, A. Malkov, A. Malygin, S. Suvorov, A. Shchukarev, A. Beljaev and V. Bykov, Thin Solid Films, 1997, 293, 91-95.

36. J. Aarik, A. Aidla, T. Uustare and V. Sammelselg, J. Cryst. Growth, 1995, 148, 268-275.

J. Aarik, A. Aidla, V. Sammelselg and T. Uustare, J. Cryst. Growth, 1997, 181, 259-264.

38. A. J. Atanacio, M. A. Alim, T. Bak, M. Ionescu and J. Nowotny, J. Am. Ceram. Soc., 2017, 100, 419-428.

A. Alonso-Tellez, R. Masson, D. Robert, N. Keller and V. Keller, J. Photochem. Photobiol. A: Chem., 2012, 250, 5865.

A. Nitta, M. Takase, M. Takashima, N. Murakami and B. Ohtani, Chem. Commun., 2016, 52, 12096-12099.

T. Berger, J. A. Anta and V. c. Morales-Flórez, J. Phys. Chem. C, 2012, 116, 11444-11455.

A. Dibenedetto, J. Zhang, M. Trochowski, A. Angelini, W. Macyk and M. Aresta, J. $\mathrm{CO}_{2}$ Util., 2017, 20, 97-104.

T. Yoshida, A. Yamaguchi, N. Umezawa and M. Miyauchi, J. Phys. Chem. C, 2018, 122, 21695-21702.

44. M. Buchalska, M. Pacia, M. Kobielusz, M. Surówka, E. Świętek, E. Wlaźlak, K. Szaciłowski and W. Macyk, J. Phys. Chem. C, 2014, 118, 24915-24924. 
45. V. Iliev, D. Tomova and L. Bilyarska, J. Photochem. Photobiol. A: Chem., 2018, 351, 69-77.

46. M. Wang, K. Han, S. Zhang and L. Sun, Coord. Chem. Rev., 2015, 287, 1-14.

47. L. Gao, Y. Li, J. Ren, S. Wang, R. Wang, G. Fu and Y. Hu, Appl. Catal. B: Environ., 2017, 202, 127-133.

48. K. L. Orchard, D. Hojo, K. P. Sokol, M.-J. Chan, N. Asao, T. Adschiri and E. Reisner, Chem. Commun., 2017, 53, 1263812641.

49. P. Karthik, R. Vinoth, P. Selvam, E. Balaraman, M. Navaneethan, Y. Hayakawa and B. Neppolian, J. Mater. Chem. A, 2017, 5, 384-396.

50. J.-L. Shi, H. Hao, X. Li and X. Lang, Catal. Sci. Technol., 2018, 8, 3910-3917.

51. M. Hidalgo, G. Colón and J. A. Navio, J. Photochem. Photobiol. A: Chem., 2002, 148, 341-348.

52. P. K. Jain, K. S. Lee, I. H. El-Sayed and M. A. El-Sayed, J. Phys. Chem. B, 2006, 110, 7238-7248.

53. C. J. Murphy, T. K. Sau, A. M. Gole, C. J. Orendorff, J. Gao, L. Gou, S. E. Hunyadi and T. Li, J. Phys. Chem. B, 2005, 109, 13857-13870.

54. N. Cheng, J. Tian, Q. Liu, C. Ge, A. H. Qusti, A. M. Asiri, A. O. Al-Youbi and X. Sun, ACS Appl. Mater. Interfaces 2013, 5, 6815-6819.

55. E. Kowalska, O. O. P. Mahaney, R. Abe and B. Ohtani, Phys. Chem. Chem. Phys., 2010, 12, 2344-2355.

56. O.-O. Prieto-Mahaney, N. Murakami, R. Abe and B. Ohtani, Chem. Lett., 2009, 38, 238-239.

57. M. V. Dozzi, L. Prati, P. Canton and E. Selli, Phys. Chem. Chem. Phys., 2009, 11, 7171-7180.

58. V. A. Lebedev, D. A. Kozlov, I. V. Kolesnik, A. S. Poluboyarinov, A. E. Becerikli, W. Grünert and A. V. Garshev, Appl. Catal. B: Environ., 2016, 195, 39-47.

59. B. Liu, X. Zhao, J. Yu, I. P. Parkin, A. Fujishima and K. Nakata, J. Photochem. Photobiol. C: Photochem. Rev., 2019, 39, 157.

60. X. Li, W. Zhang, W. Cui, J. Li, Y. Sun, G. Jiang, H. Huang, Y. Zhang and F. Dong, Chem. Eng. J., 2019, 370, 1366-1375.

61. W. Cui, L. Chen, J. Li, Y. Zhou, Y. Sun, G. Jiang, S. Lee and F. Dong, Appl. Catal. B: Environ., 2019, 253, 293-299.

62. M. Heikkilä, E. Puukilainen, M. Ritala and M. Leskelä, J. Photochem. Photobiol. A: Chem., 2009, 204, 200-208.

63. R. Pheamhom, C. Sunwoo and D.-H. Kim, J. Vac. Sci. Technol., A, 2006, 24, 1535-1539.

64. J.-G. Yu, H.-G. Yu, B. Cheng, X.-J. Zhao, J. C. Yu and W.-K. Ho, J. Phys. Chem. B, 2003, 107, 13871-13879.

65. N. R. Mathews, E. R. Morales, M. Cortés-Jacome and J. T. Antonio, Sol. Energy, 2009, 83, 1499-1508.

66. M. Surówka, M. Kobielusz, M. Trochowski, M. Buchalska, K. Kruczała, P. Broś and W. Macyk, Appl. Catal. B: Environ., 2019, 247, 173-181.

67. S. K. Cushing, F. Meng, J. Zhang, B. Ding, C. K. Chen, C.-J. Chen, R.-S. Liu, A. D. Bristow, J. Bright and P. Zheng, ACS Catal., 2017, 7, 1742-1748.

68. S. Selcuk, X. Zhao and A. Selloni, Nature Mater., 2018, 17, 923. 